\title{
Are nursing led protocols evolving with new automated mechanical ventilation weaning strategies: A systematic review
}

\author{
Brad Chesham $^{1,2 *}$ \\ ${ }^{1}$ Post Graduate Cardiothoracic Nursing, Australian Catholic University, Sydney, Australia \\ ${ }^{2} \mathrm{Msc}$ Critical Care, Anglia Ruskin University, Cambridge, UK
}

\begin{abstract}
It is known that serious physiological and psychological consequences can arise when prolonged ventilation impacts patients. New automated weaning systems may decrease the duration of mechanical ventilation and ICU length of stay. This is a new introduction to ICU weaning plans which only relatively recently have aligned with specific weaning protocols to improve outcomes.

The aim of this systematic review was to compare automated weaning against protocol based weaning. The second objective was to discover how nurses are getting involved and what is their application to the new technology?

A systematic literature search was carried out using electronic databases as well as other sources including Google scholar. A detailed inclusion and exclusion criteria was constructed and applied for screening and the quality of the included studies was thoroughly assessed. 3 studies met the associated criteria for analysis totalling a patient population of 284 .

Automated ventilator weaning proved to be beneficial over protocol based weaning. The results showed an improved outcome towards a decrease in mechanical ventilation and in ICU length of stay. No immediate impact was noted on hospital length of stay or ICU mortality. Cultural and organisational variables were noted throughout the data, generating notable heterogeneity. The decision-making process related to extubation, staffing, compliance with the delivery of the protocols and medical cover and structure across the ICU were revealed to impact data. In no study did nursing play a primary role.

In conclusion automated ventilation is beneficial over protocol based weaning. This benefit is limited and the lack of strength within the evidence results in minimal external validity. It was determined that nursing has a role to play within the process of weaning but has not yet thoroughly investigated the new technology and its possible applications.
\end{abstract}

\section{What is already known about the topic}

Greater than $30 \%$ of patients when critically ill, depend upon ICU admission and the facilitation of mechanical ventilation. Various published guidelines introduce the importance of weaning by stating that $40-45 \%$ of the time medical patients spend on a ventilator is during the discontinuation process. It is generally a life saving treatment but does come with a heavy economic burden. As these costs and complications are substantial, discontinuation at the earliest possible moment is imperative. What has been proved recently is that automated ventilation is making progress in improving weaning strategies.

\section{What this paper adds}

The implications for this analysis would be that there is justification for further investigation of alternative weaning methods against a strictly defined weaning protocol. This could help to introduce the effect of quality bedside nursing or even the multidisciplinary team into such protocols. The ability to produce a strict weaning protocol that is composed utilising an automated ventilator and delivered by a multidisciplinary team is yet to exist. All such features have individually been able to provide a benefit and it would be of great interest to the author to see how the combination would take effect. After the analysis of this data such studies must be well designed and adequately powered.
They should also attempt to compare the new technology or a more intimate application of nursing to the weaning process and finally a summative analysis to examine costing's related to each group.

\section{Introduction}

In this modern era of healthcare delivery where governments emphasize an ethos of doing more for less the challenges before us in intensive care are complex. Weaning from mechanical ventilation is one of these challenges. It is being tackled globally but are we getting any closer to finding answers to the best protocols or better still, is the technology advancing enough to make such protocols redundant?

The work undertaken towards the suspension of mechanical ventilation is known as weaning. It encompasses the process of liberating the patient off mechanical ventilation and removing the endotracheal tube [1]. This is a key component of a patient's journey

Correspondence to: Brad Chesham, Post Graduate Cardiothoracic Nursing, Australian Catholic University, Sydney, Australia, Msc Critical Care, Anglia Ruskin University, Cambridge, UK, E-mail: bradley.chesham@gmail.com

Received: December 23, 2017; Accepted: January 10, 2018; Published: January 13,2018 
through an intensive care admission and impacts all mechanically ventilated patients who survive ICU. Bendixin, et al. [2] stated within the first text on mechanical ventilation "To know the proper timing and rate of weaning from the respirator requires considerable judgment and experience. As a rule, weaning should start as soon as possible". We have moved along way from here but even with modern evidenced based practice, prolonged ventilation continues to trouble our patient population within ICU [3]. Due to this importance in outcomes, weaning perseveres as a key focus for clinical debate with many researchers understandably searching for the best method for executing respiratory care towards extubation.

Greater than $30 \%$ of patients when critically ill, depend upon ICU admission and the facilitation of mechanical ventilation [4]. Guidelines published in the CHEST journal by MacIntyre [5] introduce the importance of weaning by stating $42 \%$ of the time medical patients spend on a ventilator is during the discontinuation process. It is generally a life saving treatment but does come with a heavy economic burden. As these costs and complications are substantial, discontinuation at the earliest possible moment is imperative. Kite-Powell, et al. [6] and Grap, et al. [4] both indicated that the economic burden for a patient in an intensive care unit is approximately 4 times greater than costs for a patient who is not and a high proportion of the extra expenditure is associated to mechanical ventilation. For further perspective, Chalfin and Rizzo [7] outlined that the costs associated with medical care in ICU in the United States were estimated to be $\$ 81.7$ billion, accounting for $13.4 \%$ of hospital costs and $0.66 \%$ of the national gross domestic product.

\section{Objectives}

An immediate aim for this review was to compare automated weaning against protocol based weaning. The second objective is to discover how nurses are getting involved? What is their application to the new technology? It is possible that the new technology is rolling out and being implemented without incorporated protocols and little documented evidence of how nursing impacts patients exposed to the technology. Articles will be examined to analyse if the broader elements of ICU care are introduced into the investigative process or is it that the scientific physiological parameters are simply being measured to assess the quality of the automation.

\section{Methods}

\section{Participants}

For inclusion into this review studies must have been written in English. Eligible studies included age groups over 16 years old excluding paediatric patients. All ethnic groups have been included with no exceptions. Inclusion focused on studies examining those patients requiring weaning from mechanical ventilation with a nursing perspective. Studies with a sole technical perspective to automated and protocol weaning have been excluded as the nature of the study was to investigate the evolution of nursing care in relation to these interventions.

\section{Selection criteria}

Predominantly the inclusion of study design will align with quantitative studies rather than qualitative due to the nature of comparable assessment. Outside Randomised Control Trial's other study designs will be incorporated to allow for a wider range of inclusion across the literature. The varying study designs for inclusion will include non-randomised control trials, prospective studies, case control and cross-sectional studies would also include in study design assessment. References of included articles were also being examined as potential articles of interest.

Studies were excluded if they were published as short reports; case reports or overviews. A date must have been specified and the full text publication must have been available. The date must have ranged between 2006-2016. Outcome measures

Potential outcomes for this review would highlight that a gap in the research has been produced between weaning protocols and the new technologies and that possibly a benefit may arise by combining the two concepts. This will be examined by analysis of articles comparing automated weaning with protocol based weaning. Outcomes may also directly indicate that the new technology is beneficial and may require further support and larger studies to cement this into practice.

\section{Intervention}

When transitioning towards extubation or amidst the process of weaning, a key attribute is that the patient is able to breathe spontaneously. Put simply, once the ventilator is removed the patient will have to breathe on their own. Therefore during this transition the ventilator provides "pressure support". This allows the patient to trigger the breaths, but the machinery provides incremental assistance to the breath to support either specific tidal volumes or a level on inspiratory pressure. This level of pressure support can easily be titrated to allow assistance during weaning or the transition phase of ventilation. This is the key focus of new technologies such as SmartCare. Rose, et al. [8] describe this, "Through continuous monitoring and real time interventions, automated systems such as SmartCare, theoretically provide improved adaption of ventilatory support to patients needs when compared to clinician driven weaning". The technology manipulates the settings within the ventilator to achieve a position where the patient is ready for a spontaneous breathing trial (SBT). This is common practice amongst weaning patients in order to prepare them for extubation. If the patient is not quite prepared or titrated by the programming to the SBT, the goal is to keep the patient in a zone of respiratory comfort. Automation is likely to decrease unnecessary delays as it is less inclined to rely on the healthcare team to recognize changes in the patient's progress.

\section{Search methods}

Electronic searches: One researcher developed, conducted and refined the search strategy to collate eligible studies. Database specific searches were undertaken, and the following sources were assessed: Cochrane Central Register of controlled trails (Central); MEDLINE; EMBASE; CINAHL; Wiley Interface. The databases were searched in November 2015. Once the databases were selected they were investigated for the subject topic. Focused MeSh terms were included: Automated Weaning; Automatic weaning Mechanical ventilation; Weaning; Nursing; Protocol directed weaning; Protocol.

Searching other resources: A manual search of reference lists was also conducted of eligible studies to identify additional potential studies. Google scholar was also utilised as a search engine for prospective study inclusion. Although Google Scholar was helpful and introduced new content for analysis many articles listed were not relevant to the listed keywords. Secondarily many of the articles retrieved were simply duplicates of those found through the primary database search. Citations were downloaded into an endnote library.

Data collection and extraction: One author independently extracted: participant's characteristics, inclusion/exclusion criteria, 
weaning methods, study setting, which clinicians were involved in the weaning process study design and study outcomes. This was achieved through the use of a screening tool. Articles were screened through titles, abstract and full text if required. Randomisation methods, blinding, allocation concealment, and outcome reporting was recorded. Data extraction was not a blinded process in this review.

\section{Results}

The three studies investigated presented similar patient populations all within adult ICU environments measuring the direct comparison between automated weaning versus protocol based weaning. Across all three included studies positive effects on weaning and a reduction in overall mechanical ventilator time within ICU have been documented. The results from Liu, et al. [9] have come from a smaller single centre study and should be approached with caution. Compliance with associated regimes was not all discussed therefore the collated data could be questioned. The decision-making process for extubation at this sole Shanghai centre could be argued to be wildly different to the decision-making process in the European centres, simply on cultural preferences without discussing organisational variances. This is notable as this would directly impact on the hours taken to extubate in order to summarise the time taken to wean.

Direct application of respiratory therapists (RT) within the study by Burns, et al. [10] helps to underline the importance of vigilant skilled monitoring of ventilation in order to successfully and timely wean patients. RT's are utilised in North America but not across Europe, Asia and Australia where the ventilator management of intubated patients is undertaken primarily by bedside nursing staff. Inclusion of nursing interventions and compliance within this context must be made due to the front-line nature of nursing within ICU. Nurses within the ICU environment primarily undertake minute-by-minute assessment of physiological parameters to maintain patient safety. Wider management of the ICU patient includes concepts such as sedation, neurological assessment, fluid balance, timely administration of antibiotics and analgesia and administration of vasoactive drugs. These are all vital clinical nursing management components of the mechanically ventilated weaning patient.

Only within the most recent study by Burns, et al. [10] does an author begin to accommodate more than the physiological parameters when measuring the variables that impact on weaning. Sedation score was directly measured within the primary outcome for the study. The nursing staff within the ICU environment solely administered and managed this component of the study.

\section{Risk of bias}

Allocation (selection bias): Lellouche, et al. [11] and Burns, et al. [10] communicated group allocation through electronic mail. Whilst allocation within Liu, et al. [9] was conducted by the researcher.

Blinding (performance and detection bias): Blinding was not undertaken across all three studies due to the intervention being undertaken. Clinicians were therefore aware from beginning to end of treatment which group the patient was allocated. This omission makes it possible for the introduction of bias through the clinical staff either by conscious or unconscious decisions or actions during the study [9].

Incomplete outcome data (attrition bias): The largest trial showed an overall attrition of $14 \%$. An initial 1014 patients were eligible and a final 144 patients enrolled and randomized [11]. For any study to maintain external validity such numbers would need to be improved before data could be generalized. The positive rationale to this is that a large proportion of patients improved their clinical condition requiring a shorter weaning time and therefore stepped outside the trials inclusion criteria.

Selective reporting (reporting bias): It has been noted that outcome reporting for all trials was completed. The study by Burns, et al. [10] presented a study were the outcome of automated weaning compared with protocol weaning was a secondary outcome. The primary assessment was the compliance with the associated protocols. This was clearly presented through data analysis.

\section{Discussion}

Based on the collated data this review has demonstrated that automated systems can reduce weaning time. Interpretation of the results for clinical relevance remains challenging. Very little sub group analysis has been undertaken for example if the cohort of patients were to be medical ICU with Smartcare in relation to "easy to wean" post surgical patients with a variable system of PAV+. Little evidence is obtainable in relation to such systems as the technology is new and would be an area for future research to focus on. Throughout the studies at no time did the protocol weaning or dependant variable impact on the effect automated systems had on the duration of weaning. Within secondary outcomes automated systems reduced total ventilation time, ICU length of stay, need for tracheostomy and incidence of mechanical ventilation $>21$ days. Little evidence across the included studies showed an effect on mortality or overall hospital length of stay.

The interpretation of the data within this review has aligned with that of previous research. Previous systematic reviews by Rose, et al. [8], and Burns, et al. [12] have endeavoured to compare automated weaning with protocol based weaning, as was the primary outcome for this review. Burns, et al. [12] concluded that compared with nonautomated weaning strategies, weaning with SmartCare decreased weaning time, time to successful extubation, length of ICU stay and proportions of participants requiring prolonged ventilation for longer than seven days. Rose, et al. [8] stated that based on data from 21 trials totaling 1676 participants, utilization of an automated closed loop system may result in a reduction in the duration of weaning, ventilation and ICU stay. This directly aligns with all 3 trials analyzed that stated their primary outcomes to be a reduction in weaning and ventilation time along with ICU length of stay. Parallel to this all trails within this review were found to have a negligible effect on ICU mortality or hospital length of stay. This again corroborates with the literature $[8,12]$.

After the analysis of the included papers it can be determined that nursing is only currently playing a very small role within the introduction of automated weaning. Studies such as that by Brook, et al. [13], Danckers, et al. [14], Grap, et al. [4], Marelich, et al. [15], Tonnelier, et al. [16] have repeatedly demonstrated results that suggest nursing led or multidisciplinary weaning protocols are highly effective means of improving care, even in university ICUs where physicians are present 24 hours a day. It is clear from the search that no nursing led protocols are available that include automated weaning.

\section{Strengths and limitations of the review}

In this systematic review the trials had moderate methodology and a low to moderate risk of bias. Overall the quality of the evidence was low to moderate. Heterogeneity ensures that any results from the review should be interpreted with caution. The author has gone to great lengths to ensure the quality of the primary studies were sufficient to build upon for the remaining data analysis. Bias was closely examined 
and documented. Although blinding is near impossible due to the application of this specific intervention, certain efforts were made to blind investigators. No graphical analysis is available. The narrative synthesis has allowed an accurate representation of the data for the review. Garg, et al. [17] has described the most common flaws among systematic reviews to be a failure to assess the methodological quality of included primary studies and a failure to avoid bias in study inclusion. The strengths of the review are also supported by the quality of the initial search undertaken by the author. This has allowed a thorough screening process to be undertaken even though only three articles remained the inclusion and exclusion criteria were rigorously applied to provide suitable data.

An immediate known limitation is the lack of evidence in regard to papers included for analysis. A resultant affect is that a meta-analysis has not been performed and heterogeneity is present. Although by examining the topic a clinical pathway or clinical need for such papers focusing on nursing and its application to automated ventilation is a possible result. It was initially believed that the wider focus of protocols and the use of automated ventilators would present suitable amounts of data due to the scoping study completed for this review. This unfortunately did not develop.

\section{Clinical implications}

The implications for this analysis would be that there is justification for further investigation of alternative weaning methods against a strictly defined weaning protocol. This could help to introduce the effect of quality bedside nursing or even the multidisciplinary team into such protocols. The ability to produce a strict weaning protocol that is composed utilising an automated ventilator and delivered by a multidisciplinary team is yet to exist. All such features have individually been able to provide a benefit and it would be of great interest to the author to see how the combination would take effect. After the analysis of this data such studies must be well designed and adequately powered. They should also attempt to compare the new technology or a more intimate application of nursing to the weaning process and finally a summative analysis to examine costing's related to each group.

\section{Conclusion}

Compared with protocol weaning, automated weaning strategies decreased weaning time, ICU length of stay and presented with a lower proportion of patients receiving mechanical ventilation $>21$ days. Nursing had no immediate primary role associated with the automated ventilation. Based on data from 3 trials totalling 284 patients it is reasonable to conclude that automation is favourable over protocol based weaning strategies. No increased risk was apparent due to the new intervention, however the quality of the evidence examined could have been stronger. Elements of nursing practice and weaning have been touched upon but as a new element to ventilation, nursing has a gap to breach when seeking to integrate this technology into current bedside practice. Strong evidence exists for protocol based weaning and with the new technology also making a positive impact, it is hopeful that in the immediate future new research should arise with nurses leading the way.

\section{References}

1. Boles JM, Bion J, Connors A, Herridge M, Marsh B, et al. (2007) Weaning from mechanical ventilation. Eur Respir J 29: 1033-1056. [Crossref]

2. Bendixin HH, Hedley Whyte J, Laver MB, Pontopippidan H (1965) Respiratory care St Louis: Mosby

3. Johnson P, St John W, Moyle W (2006) Long-term mechanical ventilation in a critical care unit: existing in an uneveryday world. $J$ Adv Nurs 53: 551-558. [Crossref]

4. Grap MJ, Strickland D, Tormey L, Keane K, Lubin S, et al. (2003) Collaborative Practice: Development, Implementation, and Evaluation of a Weaning Protocol for Patients Receiving Mechanical Ventilation. Am J Crit Care 12: 454-460. [Crossref]

5. MacIntyre NR, Cook DJ, Ely EW Jr, Epstein SK, Fink JB, et al. (2001) Evidence-based guidelines for weaning and discontinuing ventilatory support. Chest 120: 375S-395S. [Crossref]

6. Kite-Powell DM, Sabau D, Ideno KT, Hartgraves D, Dahlberg CGW (1996) Optimizing Outcomes in Ventilator-Dependent Patients: Challenging Critical Care Practice. Crit Care Nurs $Q$ 19: 77-90. [Crossref]

7. Chalfin D, Rizzo JA (2012) The economics of critical care: critical care clinics Pennsylvannia: WB Saunders.

8. Rose L, Schultz MJ, Cardwell CR, Jouvet P, McAuley DF, et al. (2015) Automated versus non-automated weaning for reducing the duration of mechanical ventilation for critically ill adults and children: a cochrane systematic review and meta-analysis. Critical Care 19: 1-12. [Crossref]

9. Liu L, Xu X, Yang Y, Huang Y, Liu S, et al. (2013) Computer-driven automated weaning reduces weaning duration in difficult-to-wean patients. Chin Med J 126: 18141818. [Crossref]

10. Burns KEA, Meade MO, Lessard MR, Hand L, Zhou Q, et al. (2013c) Wean Earlier and Automatically with New Technology (the WEAN Study): A Multicenter, Pilot Randomized Controlled Trial. Am J Respir Crit Care Med 187: 1203-1211. [Crossref]

11. Lellouche F, Mancebo J, Jolliet P, Roeseler J, Schortgen F, et al. 2006a. A Multicenter Randomized Trial of Computer-driven Protocolized Weaning from Mechanical Ventilation. Am J Respir Crit Care Med 174: 894-900. [Crossref]

12. Burns KE, Lellouche F, Nisenbaum R, Lessard MR, Friedrich JO (2014) Automated weaning and SBT systems versus non-automated weaning strategies for weaning time in invasively ventilated critically ill adults. Cochrane Database Syst Rev 9: CD008638. [Crossref]

13. Brook AD, Ahrens TS, Schaiff R, Prentice D, Sherman G, et al. (1999) Effect of a nursing-implemented sedation protocol on the duration of mechanical ventilation. Crit Care Med 27: 2609-2615. [Crossref]

14. Danckers M, Grosu H, Jean R, Cruz RB, Fidellaga A, et al. (2013) Nurse-driven, protocol-directed weaning from mechanical ventilation improves clinical outcomes and is well accepted by intensive care unit physicians. J Crit Care 28: 433-441. [Crossref]

15. Marelich GP, Murin S, Battistella F, Inciardi J, Vierra T, et al. (2000b) Protocol weaning of mechanical ventilation in medical and surgical patients by respiratory care practitioners and nurses: effect on weaning time and incidence of ventilator-associated pneumonia. Chest 118: 459-467. [Crossref]

16. Tonnelier JM, Prat G, Le Gal G, Gut-Gobert C, Renault A, et al. (2005) Impact of a nurses' protocol-directed weaning procedure on outcomes in patients undergoing mechanical ventilation for longer than 48 hours: a prospective cohort study with a matched historical control group. Critical Care 9: 1-7.

17. Garg AX, Hackam D, Tonelli M (2008) Systematic Review and Meta-analysis: When One Study Is Just not Enough. Clin J Am Soc Nephrol 3: 253-260. [Crossref]

Copyright: (C2018 Chesham B. This is an open-access article distributed under the terms of the Creative Commons Attribution License, which permits unrestricted use, distribution, and reproduction in any medium, provided the original author and source are credited. 